\title{
Role of transcranial sphenoidotomy in skull base surgery: classification of surgical techniques based on the surgical anatomy of the sphenoid sinus
}

\author{
Kenichiro Iwami, MD, PhD, ${ }^{1,2}$ Masazumi Fujii, MD, PhD, ${ }^{2}$ Yugo Kishida, MD, PhD, ${ }^{3}$ \\ Shinya Jinguji, MD, PhD, ${ }^{2}$ Masayuki Yamada, MD, ${ }^{2}$ Mudathir Bakhit, MBBS, ${ }^{2}$ \\ Naoki Nishio, MD, PhD, ${ }^{4}$ Yasushi Fujimoto, MD, PhD, ${ }^{4}$ Tetsuya Ogawa, MD, PhD, ${ }^{5}$ \\ Keisuke Takanari, MD, PhD, ${ }^{6}$ Yuzuru Kamei, MD, PhD, ${ }^{6}$ and Kiyoshi Saito, MD, PhD ${ }^{2}$ \\ Departments of ${ }^{1}$ Neurosurgery and ${ }^{5}$ Otorhinolaryngology, Aichi Medical University; ${ }^{3}$ Department of Neurosurgery, Japanese Red \\ Cross Nagoya Daini Hospital, Aichi; ${ }^{2}$ Department of Neurosurgery, Fukushima Medical University, Fukushima; and Departments \\ of ${ }^{4}$ Otorhinolaryngology and ${ }^{6}$ Plastic and Reconstructive Surgery, Nagoya University Graduate School of Medicine, Nagoya, \\ Japan
}

OBJECTIVE Although sphenoidotomy is more commonly performed via the transnasal approach than via the transcranial approach, transcranial sphenoidotomy (TCS) remains indispensable for en bloc resection of locally advanced sinonasal malignant tumors (SNMTs) extending to the skull base. TCS also enables transsphenoidal transposition of the temporoparietal galeal flap (TPGF) to compensate for the lack of vascularized reconstructive tissue after endoscopic transnasal skull base surgery. The objective of this study was to review the authors' surgical experience using TCS with an emphasis on the surgical anatomy of the sphenoid sinus and on the purpose of TCS. Relevant anatomy is further illustrated through cadaveric dissection and photo documentation.

METHODS The authors reviewed the records of 50 patients who underwent TCS at the Nagoya University Hospital, Fukushima Medical University Hospital, or Aichi Medical University Hospital over the course of 7 years (between January 2011 and November 2017). The authors also performed cadaveric dissection in 2 adult cadaveric skull base specimens.

RESULTS Of the 50 patients included in this study, 44 underwent craniofacial resection (CFR) for en bloc resection of SNMTs involving the anterior and/or lateral skull base, and 6 underwent transsphenoidal transposition of the TPGF flap. The authors categorized the TCS procedures according to the portion of the sphenoid sinus wall involved (i.e., superior, lateral, and superolateral). Superior sphenoidotomy was used in patients requiring anterior CFR. Lateral sphenoidotomy was further divided into 2 subtypes, with type 1 procedures performed for the transsphenoidal transpositioning of the TPGF, and type 2 procedures used in patients requiring lateral CFR. Superolateral sphenoidotomy was used in anterolateral CFR.

CONCLUSIONS TCS still represents a useful tool in the armamentarium of neurosurgeons treating central skull base lesions. The newly proposed surgical classification facilitates a profound understanding of TCS and how to incorporate this technique into clinical practice.

https://thejns.org/doi/abs/10.3171/2018.6.JNS181013

KEYWORDS transcranial sphenoidotomy; skull base; sinonasal tumor; galeal flap; sphenoid sinus; pituitary surgery

$\mathrm{S}$ PHENOIDOTOMY represents the surgical opening of the sphenoid sinus, and it is often performed transnasally at the anterior wall to create a corridor to the sellar and parasellar regions. Anatomically, transnasal sphenoidotomy can be described as "anterior sphe- noidotomy." On the other hand, the superior and lateral walls of the sphenoid sinus can be opened transcranially. Although sphenoidotomy is more commonly performed via the transnasal approach than via the transcranial approach, transcranial sphenoidotomy (TCS) is indispens-

ABBREVIATIONS CFR = craniofacial resection; eTNA = endoscopic transnasal approach; LRS = lateral recess of the sphenoid sinus; SNMT = sinonasal malignant tumor; TCS = transcranial sphenoidotomy; TPGF = temporoparietal galeal flap.

SUBMITTED April 13, 2018. ACCEPTED June 27, 2018.

INCLUDE WHEN CITING Published online November 23, 2018; DOI: 10.3171/2018.6.JNS181013. 
able for en bloc resection of sinonasal malignant tumors (SNMTs) extending to the skull base. Furthermore, TCS enables transsphenoidal transposition of the temporoparietal galeal flap (TPGF) to compensate for the lack of vascularized reconstructive tissue after endoscopic transnasal skull base surgery.

Sinonasal tumors are tumors that occur in the nasal cavity or paranasal sinuses. SNMTs are uncommon, accounting for only $3 \%$ of malignant tumors occurring in the head and neck. ${ }^{4}$ Among the many therapeutic approaches proposed for SNMT, complete resection followed by postoperative radiotherapy has been known to provide the best outcomes., ${ }^{1,2,6}$ The nasal cavity and paranasal sinuses consist mostly of air-filled spaces, which facilitates the asymptomatic growth of the tumor until an advanced stage. Locally advanced SNMTs can involve multiple structures, such as the posterior ethmoid, posterosuperior end of the nasal septum, root of the pterygoid process, and orbital apex, all of which are in contact with the bony wall of the sphenoid sinus. Thus, surgery for resection of these lesions must include TCS in order to facilitate detachment of the affected structures from the skull without touching the tumor, which helps achieve an en bloc resection with a tumor-free margin.

In the past 2 decades, rapid advancements in endoscopic transnasal approach (eTNA) techniques has led to the use of this approach in the management of skull base lesions. Skull base defects following endoscopic transnasal surgery can usually be reconstructed using a nasal mucosal flap. However, it is sometimes difficult to reconstruct large skull base defects using nasal mucosal flaps alone. Furthermore, the tissue that may serve as a nasal mucosal flap may have already become damaged due to previous surgery or radiotherapy. In such cases, we perform TCS and transsphenoidal transposition of the TPGF to provide vascular tissue to the nasal cavity. To better understand which TCS surgical technique may be adequate in individual cases, it is necessary to examine the relevance of the sphenoid sinus surgical anatomy in more detail.

In the present study, we aimed to review our recent applications of TCS and to develop a new surgical classification for this procedure based on the anatomical variations of the sphenoid sinus. Here, we also described the technical nuances of TCS and illustrate relevant anatomy through cadaveric dissection and photographic documentation.

\section{Methods \\ Patients}

We conducted a surgical anatomy and technique study through a retrospective review of the clinical charts of 50 patients who underwent TCS at the Nagoya University Hospital, Fukushima Medical University Hospital, or Aichi Medical University Hospital, in the period between January 2011 and November 2017. The requirement for informed consent for study participation was waived due to the retrospective nature of the study. Each patient underwent either craniofacial resection (CFR) for an SNMT or transsphenoidal transposition of the TPGF. CT and MRI were performed preoperatively to evaluate the anatomy of the sphenoid sinus and the lesions. On the CT scans, the anatomy of the sphenoid sinus in the sagittal plane was classified into 3 categories based on the extension of pneumatization around the sella turcica: conchal, presellar, and sellar. ${ }^{5}$ We also evaluated the extent of lateral pneumatization of the sphenoid sinus in the coronal plane. Based on the sphenoid sinus classification proposed by Wang et al., the lateral recess of the sphenoid sinus (LRS) was defined as a measurable indentation of the bone lateral to a line passing through the medial edge of the anterior end of the vidian canal and the extracranial end of the foramen rotundum. ${ }^{17}$ In the current study, sphenoid sinuses without an LRS were anatomically classified as body type, while those with an LRS were classified as lateral type. We analyzed the operative characteristics including the surgical site and purpose of TCS.

\section{Preoperative Surgical Simulation}

Before surgery, we always perform a virtual simulation based on 3D CT data to confirm the feasibility of TCS. In most patients, a 3D virtual surgical simulation was performed using Virtual Surgiscope (created by Graduate School of Information Science, Nagoya University), which employs a volume rendering method to generate a $3 \mathrm{D}$ virtual environment based on CT image data. ${ }^{8}$

\section{Transcranial Sphenoidotomy}

The general procedure for TCS was as follows. Following craniotomy, the dura was elevated from the floor of the anterior and/or middle cranial fossa to expose the bony wall of the sphenoid sinus to be opened. Cranial nerves II-VI were transected as necessary. Then, the sphenoid sinus was opened using a high-speed drill. The aperture was widened to the desired size using the drill or Kerrison rongeurs.

\section{Surgical Strategy for Handling the SNMT}

The surgical indication for CFR was a tumor involving the anterior and/or lateral skull base, with the possibility of en bloc resection. To achieve a tumor-free field, we usually plan for osteotomy adding at least $5 \mathrm{~mm}$ to the surgical margin established during preoperative simulation. ${ }^{8}$ We cut the skull base according to the preoperative plan, using the navigation system. The contraindications to surgical management of SNMTs are usually due to the difficulty of achieving en bloc resection with tumor-free margins. In our patients, such contraindications were invasion of any sphenoid sinus wall, cavernous sinus invasion, clivus invasion, and distant metastasis. ${ }^{9}$

The surgical procedure was as follows. First, the head and neck surgeons performed facial dissection or endoscopic endonasal dissection. Surgical neck dissection was also performed in patients with cervical lymph node metastases. Next, the neurosurgeons performed cranial dissection. In CFR, osteotomy was performed using a high-speed drill with a 3-mm round burr. TCS was performed to remove the tumor extending to the periphery of the sphenoid sinus. After TCS, the anterior wall and/or floor of the sphenoid sinus were drilled from the interior cavity of the sinus to achieve en bloc resection without 

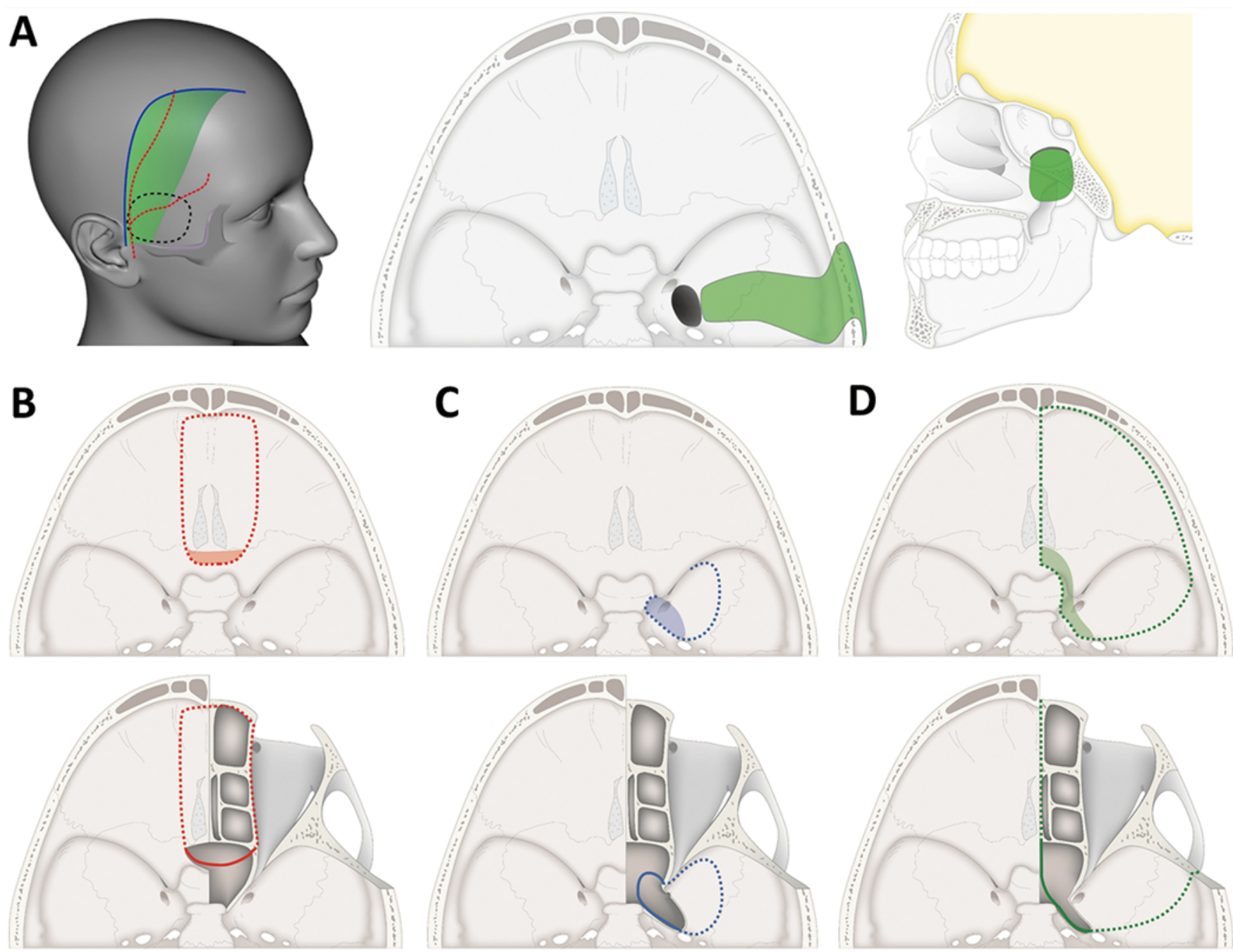

FIG. 1. Use of TCS. A: Transsphenoidal transposition of the TPGF: blue line, hemicoronal skin incision; green area, TPGF; red dotted line, superficial temporal artery; black dotted line, temporal craniotomy; black ellipse, transcranial sphenoidotomy. B-D: Classification of CFR: anterior CFR (B); lateral CFR (C); and anterolateral CFR (D). Dotted lines indicate the resection lines on the intracranial aspect of the skull base, while continuous lines indicate the resection lines on the sphenoid sinus (red, anterior CFR; blue, lateral CFR; green, anterolateral CFR). The areas colored red, blue, or green indicate the TCS in each type of CFR. Figure is available in color online only.

any exposure of the tumor. After resection of the tumor, the plastic surgeons reconstructed the defect of the cranial base using a galeal flap or a rectus abdominis myocutaneous free flap.

\section{Transsphenoidal Transposition of the TPGF}

The steps involved in TPGF transsphenoidal transposition are outlined in Fig. 1A. Through a hemicoronal skin incision, a unilateral TPGF with adequate length and width was harvested. A small temporal craniotomy was performed, followed by extradural elevation of the temporal lobe. Because the TPGF received blood supply from the superficial temporal artery, the pedicle was identified anterior to the ear. Thus, TCS was designed to allow insertion of the TPGF into the sphenoid sinus through the lateral wall, at the nearest possible distance from the flap ped- icle. The TPGF was introduced through the middle fossa extradural space and sinus opening, with caution to avoid any torsion or strangulation of the pedicle. After transnasal sphenoidotomy, the TPGF was placed into the nasal cavity and used for the reconstruction of the skull base defect.

\section{Observation of the Sphenoid Sinus in Dry Skulls}

Two adult dried skulls of unknown age or sex were used for an observational experiment. One skull had lateral-type sphenoid sinus anatomy, while the other had bodytype sphenoid sinus anatomy. Both skulls were cut along the midline sagittal plane to illuminate the interior of the sphenoid sinus with a light source and permit observation. We observed the shape of the sphenoid sinus from the middle cranial fossa, relying on the pattern of transmitted light through the sphenoid bone. 


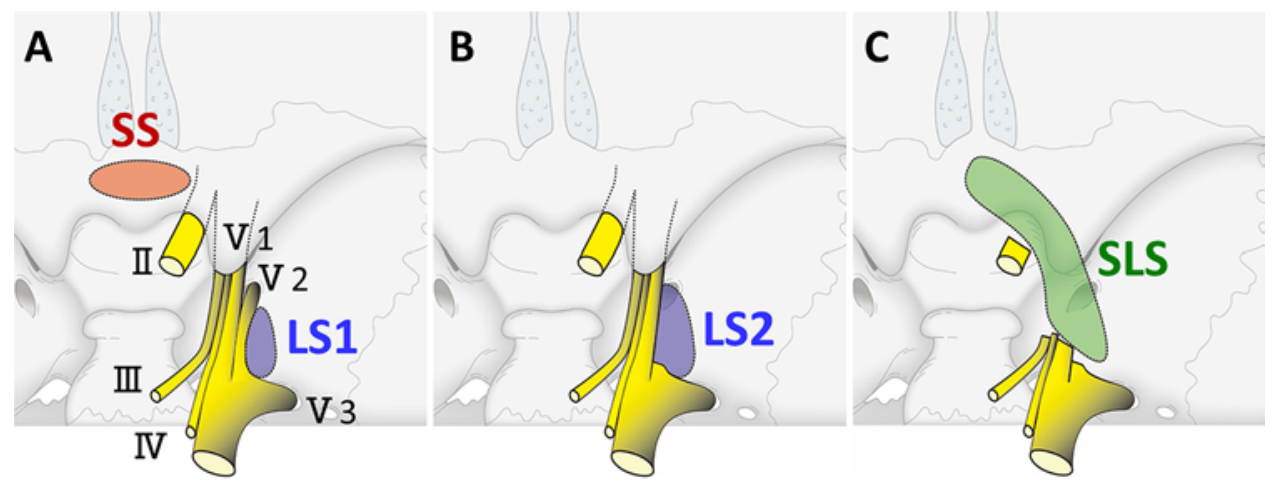

FIG. 2. Classification of TCS techniques. A: Superior sphenoidotomy (SS, red) and type 1 lateral sphenoidotomy (LS1, blue). B: Type 2 lateral sphenoidotomy (LS2, blue). C: Superolateral sphenoidotomy (SLS, green). II = optic nerve; III = oculomotor nerve; IV = trochlear nerve; V1 = ophthalmic nerve; V2 = maxillary nerve; V3 = mandibular nerve. Figure is available in color online only.

\section{Cadaveric Dissection}

We performed cadaveric dissection in 2 adult cadaveric skull base specimens, using $4 \times$ to $20 \times$ magnification. All studies were performed using an actual surgical setting.

\section{Results \\ Patient Characteristics and Application of Transcranial Sphenoidotomy}

A total of 50 patients (34 men and 16 women) were included in this study. Their mean age at surgery was 58.98 years (range $8-81$ years). In this series, TCS was used in CFR of SNMTs $(\mathrm{n}=44)$ and for transsphenoidal transposition of the TPGF $(n=6)$. Among the 44 patients who underwent CFR of SNMTs, the histopathology results revealed squamous cell carcinoma $(\mathrm{n}=20 ; 45.5 \%)$, olfactory neuroblastoma $(\mathrm{n}=8 ; 18.2 \%)$, undifferentiated carcinoma $(n=4 ; 9.1 \%)$, adenocystic carcinoma $(n=3$; $6.8 \%)$, cylindrical cell carcinoma $(\mathrm{n}=2 ; 4.5 \%)$, basal cell carcinoma $(n=2 ; 4.5 \%)$, and other pathologies $(n=5$; $11.3 \%)$. CFR procedures were classified as anterior, lateral, and anterolateral, based on the portion of the skull base involved (Fig. 1B-D). Anterior CFR was performed in patients with SNMTs involving the anterior skull base $(n=13)$. In these patients, TCS was performed to detach the posterior ethmoid and/or posterosuperior end of the nasal septum from the skull. Lateral CFR was performed in patients with SNMTs involving the middle skull base $(n=3)$. In these patients, TCS was performed to detach the root of the pterygoid process from the skull. Finally, anterolateral CFR was performed in patients with SNMT involving the orbital apex $(\mathrm{n}=28)$. In these patients, TCS was performed to detach the orbital apex from the skull. The posterior ethmoid and/or the root of the pterygoid process were also removed with orbit, according to the extent of the tumor.

In the 6 patients who underwent transsphenoidal transposition of the TPGF, it was not possible to use a nasoseptal flap or other intranasal flaps because such flaps were too small to cover the defect that resulted from removal of a large tumor $(n=1)$, were unavailable due to previ- ous surgery $(n=4)$, or were unavailable due to previous radiotherapy $(n=1)$. Reconstruction required the use of vascularized tissue to prevent CSF leakage. The underlying diseases were cavernous hemangioma $(n=1)$, recurrent pituitary adenoma $(n=2)$, recurrent CSF leak from the LRS $(n=2)$, and radiation necrosis of the skull base in a patient with adenocystic carcinoma $(\mathrm{n}=1)$.

\section{Classification of TCS Procedures}

Given the superolateral position of the optic canal, we defined an imaginary line parallel to its axis as the border between the superior and lateral walls of the sphenoid sinus. Based on the part of the wall chosen to open, we classified TCS into 3 types: superior, lateral, and superolateral (Fig. 2A-C).

Superior sphenoidotomy involves the surgical opening of the sphenoid planum and provides transcranial access to the sphenoid sinus from above (Fig. 2A). It does not require cranial nerve sacrifice. In the current study, superior sphenoidotomy was performed in anterior CFR.

Lateral sphenoidotomy involves the surgical opening of the lateral wall of the sphenoid sinus and provides transcranial access to the sphenoid sinus from the side. Whether or not lateral sphenoidotomy can be performed depends on the extent of lateral pneumatization of the sphenoid sinus. Upon examining 2 dried skulls (one with body-type and the other with lateral-type sphenoid sinus anatomy; Fig. 3A and B), we found that the maxillary nerve and the mandibular nerve form a lateral loop (indicated by dotted lines in Fig. 3A and B), which represents the medial limit of dural elevation in the middle cranial fossa. ${ }^{18} \mathrm{Be}-$ cause the sphenoid sinus with body-type anatomy was not pneumatized lateral to this lateral loop (Fig. 3A), it would have been difficult to open it from the middle cranial fossa without transecting the maxillary nerve (Fig. 3C). On the other hand, in the lateral-type sphenoid sinus, the LRS was pneumatized lateral to this lateral loop and therefore could be opened from the middle cranial fossa without sacrificing any cranial nerve (Fig. 3B and D). We defined type 1 lateral sphenoidotomy as the surgical opening of the LRS without sacrifice of the maxillary nerve (Fig. 2A). In the current study, type 1 lateral sphenoidotomy was per- 

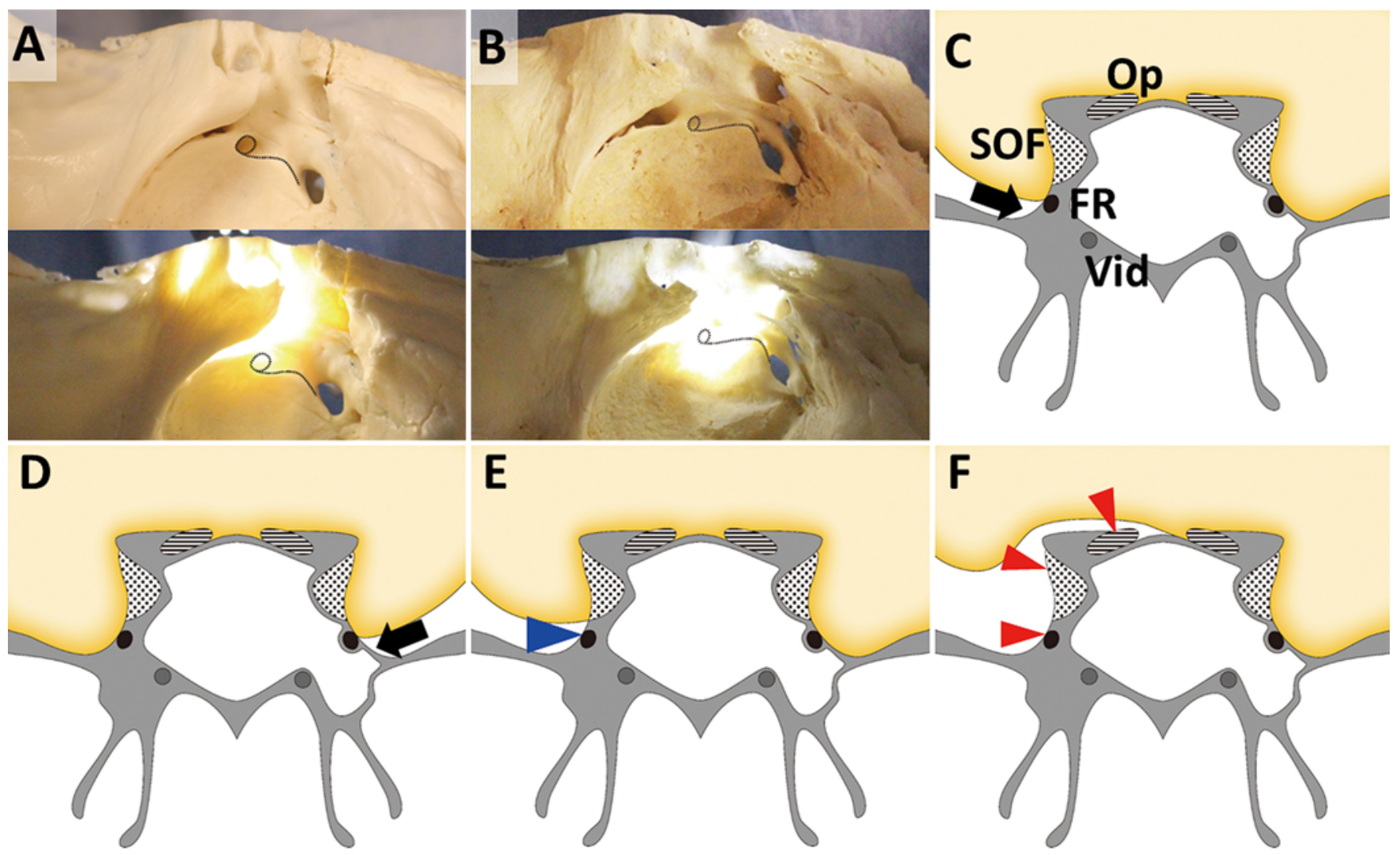

FIG. 3. Observation of the sphenoid sinus in dry skulls. A and B: The interior of the left sphenoid sinus was illuminated, revealing body-type (panel A) or lateral-type (panel B) anatomy of the sphenoid sinus. The lateral loop formed by the maxillary nerve and the mandibular nerve is indicated by dotted lines. C-F: Schematic coronal sections of sphenoid sinuses illustrating body-type pneumatization on the left side and lateral-type pneumatization on the right side. Op (stripe pattern) = optic canal, SOF (dot pattern) = superior orbital fissure, FR (black circle) $=$ extracranial end of the foramen rotundum, Vid (gray circle) $=$ anterior end of the vidian canal. In body-type anatomy, it is difficult to open the sphenoid sinus without sacrificing the maxillary nerve ( $\mathrm{C}$, black arrow). In lateral-type anatomy, the lateral recess can be opened without sacrificing the maxillary nerve (D, black arrow). Transection of the maxillary nerve enables lateral sphenoidotomy of the body-type sphenoid sinus ( $E$, blue arrowhead). By cutting the contents of the optic canal, superior orbital fissure, and foramen rotundum, it is possible to expose the superior wall and lateral wall of the sphenoid sinus consecutively ( $\mathrm{F}$, red arrowheads). Figure is available in color online only.

formed for the transsphenoidal transposition of the TPGF, with preservation of both the vidian nerve and the vidian artery (Fig. 1A). On the other hand, cutting the maxillary nerve allows for a wider lateral sphenoidotomy without additional disability in patients undergoing total maxillectomy. Thus, type 2 lateral sphenoidotomy was defined as an expanded lateral sphenoidotomy facilitated by cutting the maxillary nerve (Fig. 2B). The advantage of type 2 lateral sphenoidotomy is that it can be applied for both lateral-type and body-type sphenoid sinus anatomies (Fig. $3 \mathrm{E})$. In the current study, a type 2 lateral sphenoidotomy was used in lateral CFR.

Superolateral sphenoidotomy is a combination of superior sphenoidotomy and type 2 lateral sphenoidotomy, which provides transcranial access to the sphenoid sinus from above and from the side (Fig. 2C). Superolateral sphenoidotomy always sacrifices the contents of the optic canal, superior orbital fissure, and foramen rotundum. Thus, the superolateral wall of the sphenoid sinus can be exposed extradurally, and superolateral sphenoidotomy can be performed for almost all sphenoid sinus anatomies (Fig. 3F). In the current study, superolateral sphenoidotomy was used in anterolateral CFR.

We performed superior sphenoidotomy, type 1 lateral sphenoidotomy, type 2 lateral sphenoidotomy, and superolateral sphenoidotomy in $13,6,3$, and 28 patients, respectively. The application and classification of various transcranial sphenoidotomy procedures are outlined in Table 1.

\section{Cadaveric Dissection}

To demonstrate the feasibility and efficacy of TCS, we performed 2 cadaveric surgical anatomy studies. Figure $4 \mathrm{~A}-\mathrm{F}$ illustrates the cadaveric study demonstrating lateral sphenoidotomy and superolateral sphenoidotomy in a specimen with lateral-type sphenoid sinus anatomy (Fig. 4A). The LRS was opened just lateral to the lateral loop, without sacrificing any cranial nerve (type 1 lateral sphenoidotomy) (Fig. 4B). After transection of the maxillary nerve (Fig. 4C), the sphenoid sinus could be opened more 
TABLE 1. Applications of TCS in this study

\begin{tabular}{llc}
\hline \multicolumn{1}{c}{ Application } & Type of TCS & No. of Pts \\
\hline $\begin{array}{l}\text { Detachment of } \\
\text { 1) Posterior ethmoid \&/or posterosuperior } \\
\text { end of nasal septum }\end{array}$ & SS & 13 \\
\hline 2) Root of pterygoid process & LS2 & 3 \\
\hline 3) Orbital apex & SLS & 6 \\
\hline $2+3$ & SLS & 20 \\
\hline $1+2+3$ & SLS & 2 \\
\hline Transsphenoidal transposition of TPGF & LS1 & 6 \\
\hline
\end{tabular}

LS = lateral sphenoidotomy; LS1 = type $1 \mathrm{LS} ; \mathrm{LS} 2$ = type $2 \mathrm{LS}$; pts = patients; SLS = superolateral sphenoidotomy; $S S=$ superior sphenoidotomy; TCS = transcranial sphenoidotomy; TPGF = temporoparietal galeal flap.

widely (type 2 lateral sphenoidotomy) (Fig. 4D). Furthermore, transection of the contents of the superior orbital fissure and optic canal was performed to expose the bony wall of the sphenoid sinus more medially to the sphenoid planum (Fig. 4E), and a superolateral sphenoidotomy was performed (Fig. 4F).

Figure $4 \mathrm{G}-\mathrm{J}$ illustrates the cadaveric dissection of a skull base specimen with body-type sphenoid sinus anatomy (Fig. 4G). By drilling the lateral loop, the vidian canal was exposed, but the sphenoid sinus could not be opened (Fig. 4H). After drilling the sphenoid planum, the interior of the sphenoid sinus was illuminated using a transnasal endoscope. Upon careful examination, we confirmed the feasibility of superior sphenoidotomy but concluded that a type 1 lateral sphenoidotomy could not be performed (Fig. 4I). Even though the sphenoid sinus had body-type anatomy, the lateral wall of the sphenoid sinus could be exposed, and superolateral sphenoidotomy could be performed only after transection of the optic nerve, oculomotor nerve, trochlear nerve, ophthalmic nerve, maxillary nerve, and abducens nerve (Fig. 4J).

\section{Type of Sphenoid Sinus Pneumatization}

The technical difficulty of TCS depends mainly on the degree of sphenoid sinus pneumatization and the thickness of the bony wall of the sphenoid sinus. All 13 patients who underwent superior sphenoidotomy had sellar-type pneumatization. Of the 28 patients who underwent superolateral sphenoidotomy, 26 had sellar-type, 2 had presellar-type, and none had conchal-type pneumatization; moreover, the sphenoid planum was well pneumatized in all patients. All 6 patients who underwent a type 1 lateral sphenoidotomy had lateral-type sphenoid sinus anatomy. Among the 3 patients who underwent a type 2 lateral sphenoidotomy, one had lateral-type and two had body-type sphenoid sinus anatomy. Of the 28 patients who underwent superolateral sphenoidotomy, 15 had lateral-type and 13 had body-type sphenoid sinus anatomy.

\section{Illustrative Cases \\ Case 1}

A 72-year-old man with olfactory neuroblastoma invading the bilateral frontal lobes and posterior ethmoid bone
(Fig. 5A and B) underwent anterior CFR. Through the opening created by superior sphenoidotomy, the anterior wall and floor of the sphenoid sinus were cut (Fig. 5C-E). After performing osteotomies in the anterior cranial base, the tumor was removed in an en bloc fashion (Fig. 5F).

\section{Case 2}

A 73-year-old woman with palatine squamous cell carcinoma involving the root of the pterygoid process (Fig. 6A-C) underwent lateral CFR. A type 2 lateral sphenoidotomy provided adequate exposure to cut the sphenoid floor (Fig. 6D-F). After cutting the middle cranial base, the tumor was resected in an en bloc fashion (Fig. 6G and $\mathrm{H}$ ).

\section{Case 3}

A 60-year-old man with maxillary sinus squamous cell carcinoma involving the orbital apex and the root of the pterygoid process (Fig. 7A and B) underwent anterolateral CFR. A superolateral sphenoidotomy provided adequate exposure to cut the anterior wall and floor of the sphenoid sinus (Fig. 7C and D). After performing osteotomies in the anterior and middle cranial base, the tumor was resected in an en bloc fashion (Fig. 7E and F).

\section{Case 4}

A 46-year-old man who had developed spontaneous CSF rhinorrhea complicated by meningitis was initially treated with conservative therapy and endoscopic repair using a pedicled nasoseptal flap. However, the leak did not resolve. Because the CSF rhinorrhea was found to originate from the left LRS, we performed transsphenoidal transposition of a TPGF to cover the leak area. Following hemicoronal skin incision, the TPGF was harvested, and a small temporal craniotomy was performed (Fig. 8A). The TPGF was transposed into the sphenoid sinus through the opening created by a type 1 lateral sphenoidotomy (Fig. $8 \mathrm{~B}-\mathrm{D})$. The lateral wall of the sphenoid sinus was covered with the TPGF, and the leak immediately stopped, without the use of external drainage (Fig. 8E and F).

\section{Discussion}

The sphenoid sinus has a critical role in skull base surgery because it serves as an anatomical buffer zone or communication between the intracranial and extracranial space. We use TCS in conventional and new applications, including CFR of SNMTs and transsphenoidal transposition of TPGFs. In the 50 patients included in this study, superolateral sphenoidotomy was the most common type of TCS, which reflects the fact that the maxillary sinus is the most common primary site of SNMTs in Japanese patients (Report of Head and Neck Cancer Registry of Japan Clinical Statistics of Registered Patients in 2002, available at http://www.jshnc.umin.ne.jp/pdf/2002syourei_houkoku. pdf).

In our institutions, CFR has been performed to treat SNMTs extending to the skull base since 1992. ${ }^{9,13}$ Because CFR employs transcranial and transfacial/transnasal approaches, the neurosurgeon plays an important role in the treatment of patients undergoing CFR. While SNMTs are uncommon, some patients have SNMT extending to the 

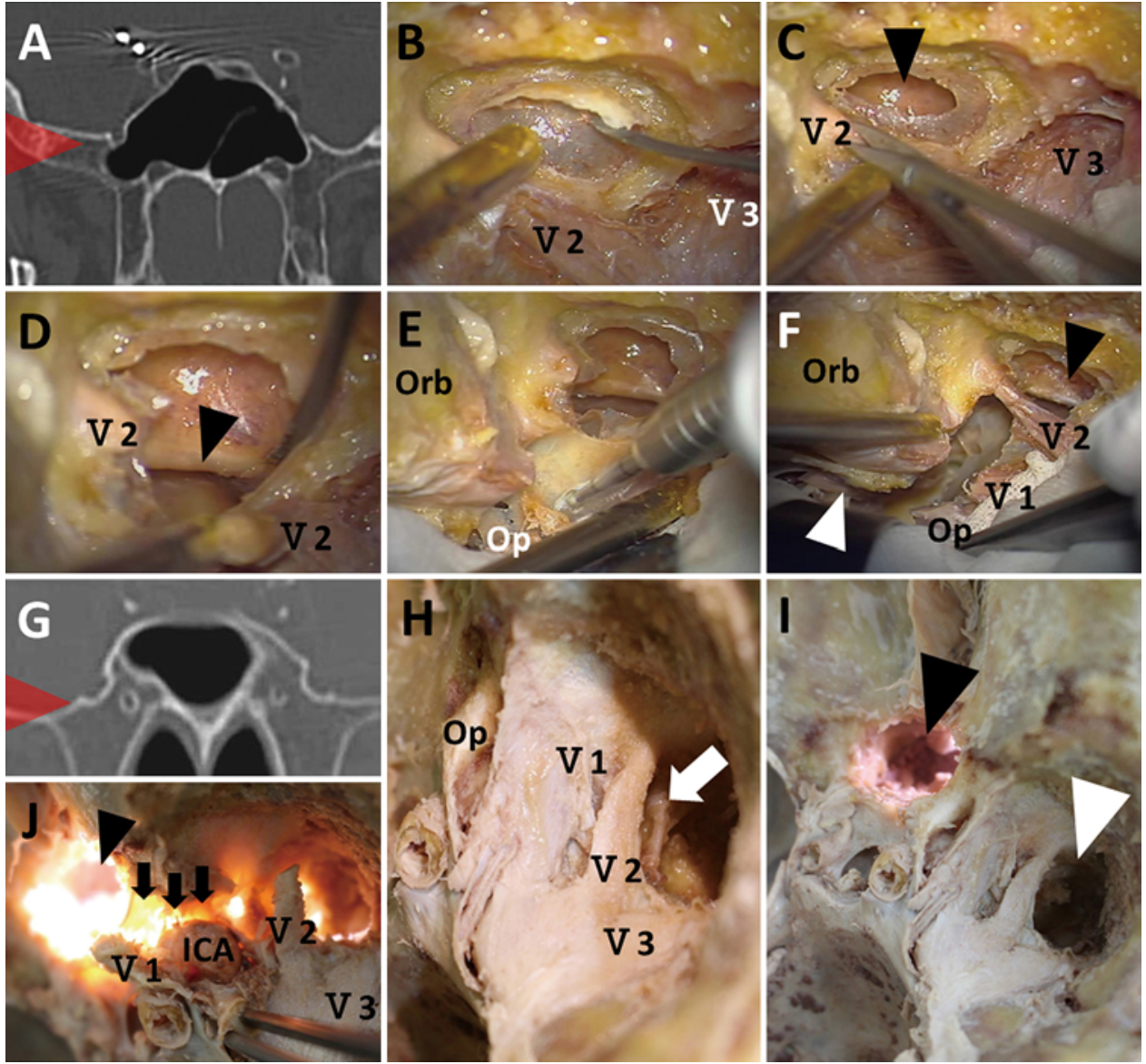

FIG. 4. Cadaveric studies illustrating the usefulness of different transcranial sphenoidotomy techniques. A-F: Cadaveric study demonstrating lateral and superolateral sphenoidotomy in a specimen with lateral-type anatomy of the sphenoid sinus. Panel $A$ shows a coronal CT scan of the cadaver head. The cadaveric study was performed on the right side (red arrowhead). After drilling the lateral loop, the lateral wall of the sphenoid sinus was thinned and removed (B). The mucosa of the lateral recess was opened (C, black arrowhead). After transecting the maxillary nerve, the sphenoid sinus could be opened more widely (D). The black arrowhead indicates a bony prominence overlaying the vidian canal. After transecting the contents of the superior orbital fissure and optic canal, the bony wall of the sphenoid sinus was exposed more medially to the sphenoid planum (E). A superolateral sphenoidotomy was performed $(F)$. The white arrowhead indicates the sphenoid planum; the black arrowhead indicates the lateral recess of the sphenoid sinus. G-J: Cadaveric study demonstrating the infeasibility of type 1 lateral sphenoidotomy and the feasibility of superior and superolateral sphenoidotomy in a specimen with body-type anatomy of the sphenoid sinus. Panel $\mathrm{G}$ shows a coronal CT scan of the cadaver head. The cadaveric study was performed on the right side (red arrowhead). By drilling the lateral loop, the vidian canal was exposed $(\mathrm{H}$, white arrow), but the sphenoid sinus was not opened. After drilling the sphenoid planum, the interior of the sphenoid sinus was illuminated with the transnasal endoscope to confirm that the superior wall was opened (l, black arrowhead), but the lateral wall was not opened (white arrowhead). The opening of the superior wall (J, black arrowhead) was extended laterally, reaching the lateral wall (black arrows), and anteriorly to the internal carotid artery after transecting the contents of the superior orbital fissure and optic canal. ICA = internal carotid artery; Op = optic nerve; Orb = orbit; V1 = ophthalmic nerve; $\mathrm{V} 2$ = maxillary nerve; $\mathrm{V} 3=$ mandibular nerve. Figure is available in color online only.

periphery of the sphenoid sinus. To achieve en bloc resection of locally advanced SNMTs, it is necessary to transect the bony skull base around the sphenoid sinus, which represents the most technically challenging part of the surgery. To avoid tumor exposure and complications, it is necessary to cut the anterior wall and floor of the sphenoid sinus accurately and safely from the inside of the sphenoid sinus, ${ }^{8}$ which can only be achieved if a sufficiently wide opening can be created into the sphenoid sinus transcranially, allowing a wide view of the interior of the sphenoid sinus.

Before the development of eTNA techniques, transcra- nial surgery was the standard treatment for benign tumors in the central skull base. ${ }^{14,15}$ When performing a piecemeal resection of benign tumors, TCS provides safe access to tumors involving the sphenoid sinus, sella, and upper clivus. With the rapid development of eTNA-based protocols, there are fewer opportunities to use a transcranial approach and TCS in patients with benign lesions. The shift from a transcranial approach to eTNA has led to an increasing need for endonasal skull base reconstruction, which typically requires the use of well-vascularized tissue, because failure of reconstruction can lead to fatal complications. The TPGF is a pedicled scalp flap with well-established 

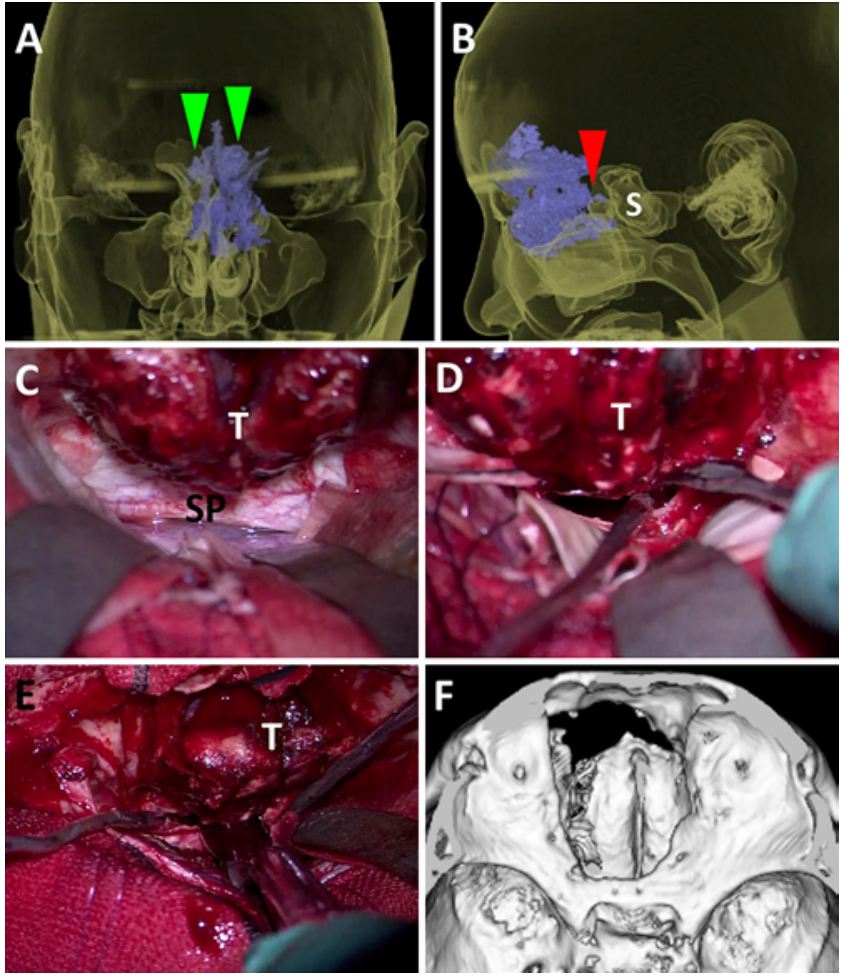

FIG. 5. Case 1: CT images and photographs. A and B: Preoperative 3D CT showing olfactory neuroblastoma (purple) invading the bilateral frontal lobes (green arrowheads) and posterior ethmoid (red arrowhead). $\mathrm{S}=$ sphenoid sinus. C-E: Intraoperative microscopic photographs. The anterior cranial base was exposed subdurally, leaving a $1-\mathrm{cm}$ margin of normal brain tissue (C). A superior sphenoidotomy was performed (D). Through the opening created by superior sphenoidotomy, parts of the intersphenoid septum, anterior wall of the sphenoid sinus, and sphenoid floor were cut using a drill and curved chisel $(E) . T=$ tumor covered with normal brain tissue; SP = dura mater of the sphenoid planum. F: Postoperative 3D CT image. Figure is available in color online only.

utility in transcranial surgery. ${ }^{7}$ TCS enables the transsphenoidal transposition of the TPGF, which augments endonasal skull base reconstruction when nasal mucosal flaps are inadequate or unavailable. Some reports have described the effectiveness of transpterygoid or transmaxillary transposition of temporoparietal fascia flaps. ${ }^{3,10-12}$ Compared with these existing methods, transsphenoidal transposition can provide a more anatomically direct route to the sphenoid sinus and upper clivus. In patients with lateral-type sphenoid sinus anatomy, the lateral loop is an area of thin bone where the sphenoid sinus can be opened easily. Adjusting the size of the aperture helps avoid strangulation of the TPGF pedicle. Because the TPGF can provide sufficient coverage of the lateral recess of the sphenoid sinus, this method is suitable for the treatment of CSF leaks from the LRS, which is the most common site of spontaneous sphenoid CSF leak..$^{19,20}$

A significant disadvantage is that transsphenoidal transposition can only be performed in patients with lateraltype sphenoid sinus anatomy. Additionally, transsphenoidal transposition requires craniotomy and temporal lobe retraction. Intracranial procedures must be performed
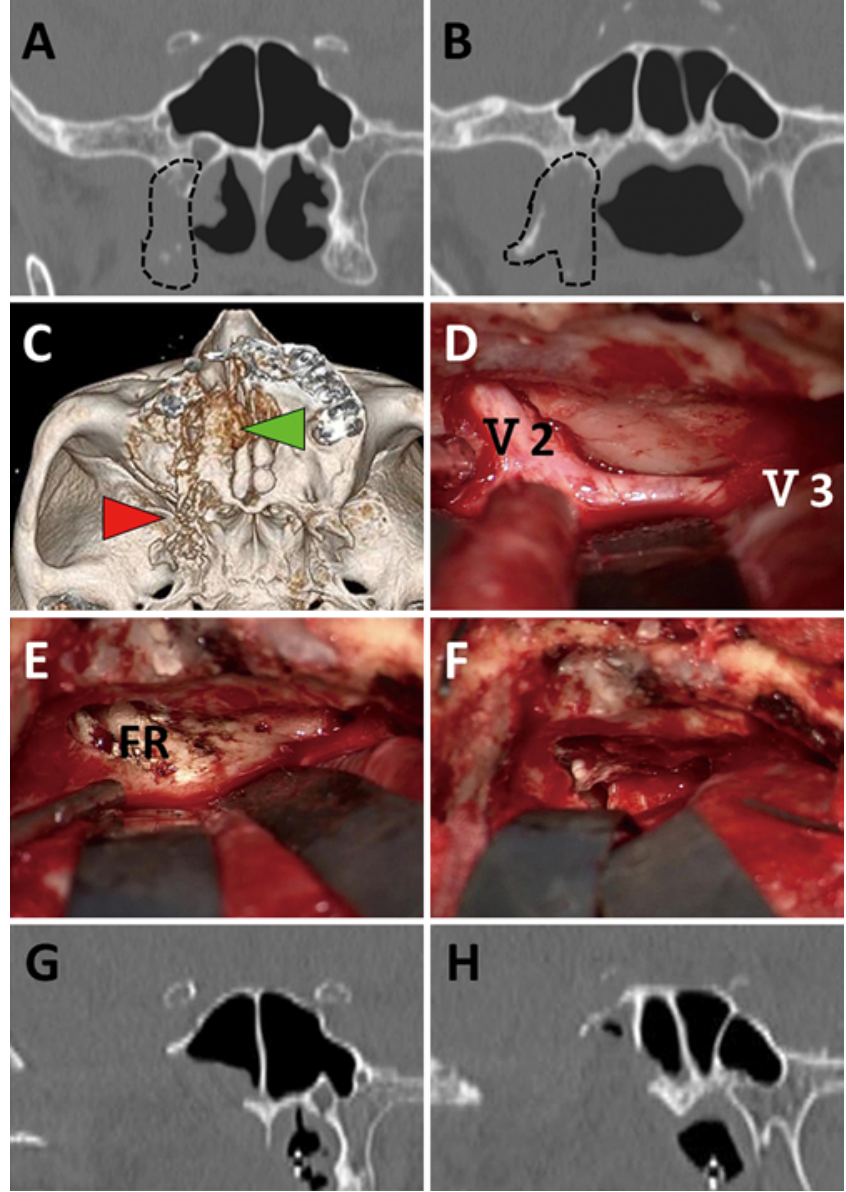

FIG. 6. Case 2: CT images and photographs. A and B: Preoperative coronal CT scans. The dotted line in each image delimits the area with bone erosion of the pterygoid process. C: Preoperative 3D CT image showing bone erosion of the right half of the hard palate (green arrowhead) and pterygoid process (red arrowhead). D-F: Intraoperative microscopic photographs. The lateral loop was exposed (D). The maxillary nerve was transected at the foramen rotundum (E). A type 2 lateral sphenoidotomy was performed (F). G and H: Postoperative coronal CT scans. FR = foramen rotundum; V2 = maxillary nerve; $\mathrm{V} 3$ = mandibular nerve. Figure is available in color online only.

with great care not to damage the central nervous system during transsphenoidal transposition of the TPGF.

Although most adults are expected to have a wellpneumatized sphenoid sinus, the degree of pneumatization varies, and careful preoperative evaluation is necessary to determine the optimal TCS technique. Using preoperative 3D simulation is helpful in confirming the feasibility of TCS. ${ }^{8}$ In patients with a well-pneumatized sphenoid sinus, a wide opening can be easily created by drilling the thin region of the bony wall. Landmarks such as the intersphenoid septum and bony indentations in the sphenoid sinus can be used to accurately determine the resection line during CFR. While poor pneumatization of the sphenoid sinus is rare in adults, it may preclude TCS. In patients with a poorly pneumatized sphenoid sinus, using the navigation system will improve the safety of CFR because there are no landmarks in the thick sphenoid bone, and inaccurate 

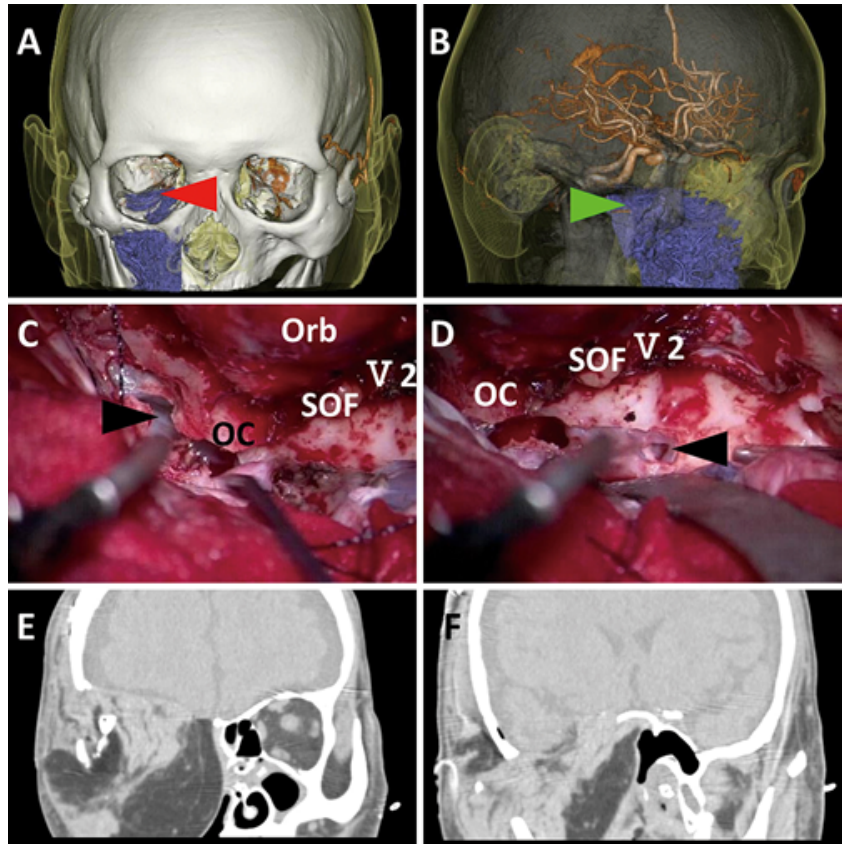

FIG. 7. Case 3: CT images and photographs. A and B: Preoperative 3D CT images showing maxillary sinus carcinoma (purple) invading the orbital apex (red arrowhead) and root of the pterygoid process (green arrowhead). C and D: Intraoperative microscopic photographs showing the opened sphenoid planum (black arrowhead in panel C) and the LRS (black arrowhead in panel D). E and F: Postoperative coronal CT scans. Orb = orbit; OC = cut end of the optic canal; SOF = cut end of the superior orbital fissure; V2 = cut end of the maxillary nerve. Figure is available in color online only.

osteotomy around the sphenoid bone can lead to tumor exposure or fatal complications. Body-type sphenoid sinus anatomy is a contraindication for transsphenoidal transposition of the TPGF due to the difficulty of performing a type 1 lateral sphenoidotomy. Vaezi et al. reported that $54 \%$ of evaluated patients had LRS pneumatization lateral to the foramen rotundum in the coronal plane. ${ }^{16}$ Transpterygoid or transmaxillary transposition is recommended in patients with poor sphenoid sinus pneumatization..$^{3,12}$

In this study, we focused on the surgical classification and application of TCS. The primary limitation of this surgical anatomy and technique study is that we did not evaluate the clinical outcome and complications. The main reason we could not perform such analyses is that this study included a relatively small series of patients with a wide range of pathologies and treatment plans. Further studies should include larger series of patients with longer follow-up in order to evaluate the efficacy and safety of TCS procedures in the management of skull base lesions.

\section{Conclusions}

We reviewed 50 cases involving TCS. Based on the surgical anatomy and technique, we proposed a new classification of TCS, with an emphasis on the specific applications of each particular technique. Considering that TCS is not frequently used, a systematic classification is required to understand how to best incorporate this procedure in clinical
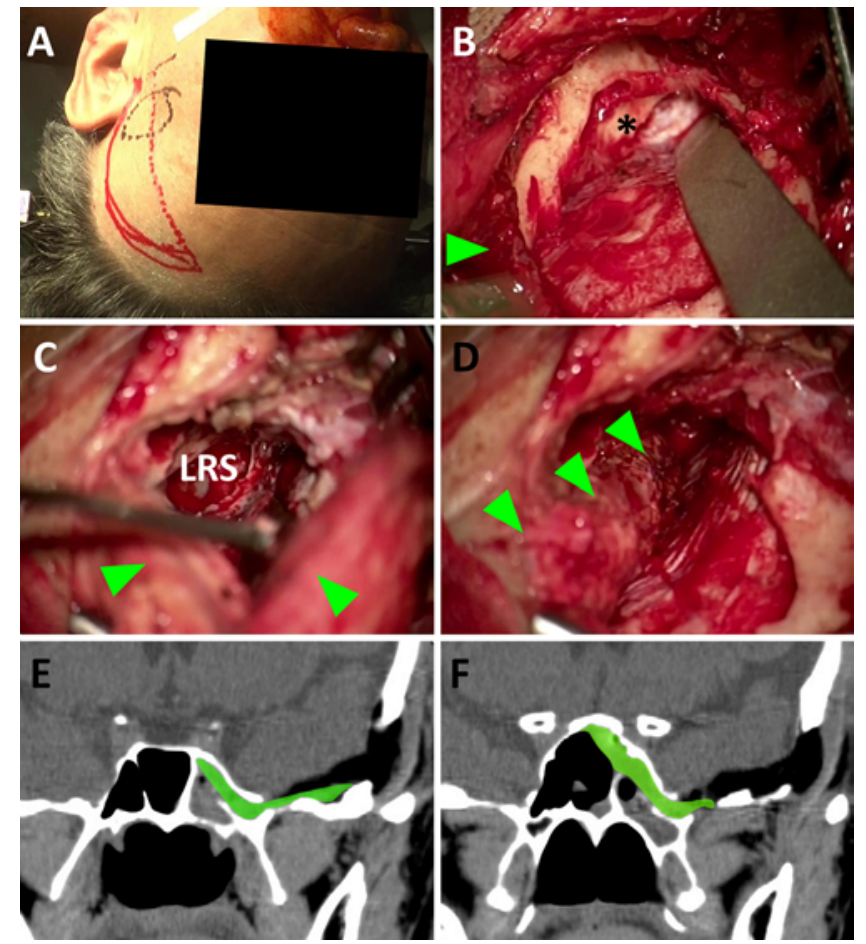

FIG. 8. Case 4: intraoperative photographs and postoperative coronal CT scans. A: The design of the TPGF (red) and the craniotomy (black). $B$ and C: Following temporal craniotomy, a type 1 lateral sphenoidotomy was performed. D: The TPGF was transposed into the sphenoid sinus. $E$ and $F$ : The lateral wall of the sphenoid sinus was covered with the TPGF (green). Green arrowheads indicate the TPGF; asterisk indicates lateral loop. Figure is available in color online only.

practice. Even in the era of endoscopic skull base surgery, TCS remains indispensable for managing skull base lesions.

\section{Acknowledgments}

We thank Hiroyuki Yaginuma, MD, PhD, Department of Neuroanatomy and Embryology, Fukushima Medical University, for constant support. This work was supported by Grant-in-Aid for Young Scientists (B) 15K19976 to Dr. Iwami from the Japan Society for the Promotion of Science.

\section{References}

1. Blanco AI, Chao KS, Ozyigit G, Adli M, Thorstad WL, Simpson JR, et al: Carcinoma of paranasal sinuses: long-term outcomes with radiotherapy. Int J Radiat Oncol Biol Phys 59:51-58, 2004

2. Dulguerov P, Jacobsen MS, Allal AS, Lehmann W, Calcaterra T: Nasal and paranasal sinus carcinoma: are we making progress? A series of 220 patients and a systematic review. Cancer 92:3012-3029, 2001

3. Fortes FS, Carrau RL, Snyderman CH, Kassam A, Prevedello D, Vescan A, et al: Transpterygoid transposition of a temporoparietal fascia flap: a new method for skull base reconstruction after endoscopic expanded endonasal approaches. Laryngoscope 117:970-976, 2007

4. Grant RN: The incidence of and mortality from cancer in the United States. Prog Clin Cancer 4:34-47, 1970

5. Hammer G, Radberg C: The sphenoidal sinus. An anatomi- 
cal and roentgenologic study with reference to transsphenoid hypophysectomy. Acta Radiol 56:401-422, 1961

6. Hoppe BS, Stegman LD, Zelefsky MJ, Rosenzweig KE, Wolden SL, Patel SG, et al: Treatment of nasal cavity and paranasal sinus cancer with modern radiotherapy techniques in the postoperative setting-the MSKCC experience. Int J Radiat Oncol Biol Phys 67:691-702, 2007

7. Ito E, Watanabe T, Sato T, Ichikawa M, Oda K, Matsumoto Y, et al: Skull base reconstruction using various types of galeal flaps. Acta Neurochir (Wien) 154:179-185, 2012

8. Nishio N, Fujii M, Hayashi Y, Hiramatsu M, Maruo T, Iwami $\mathrm{K}$, et al: Preoperative surgical simulation and validation of the line of resection in anterolateral craniofacial resection of advanced sinonasal sinus carcinoma. Head Neck 39:512_ 519,2017

9. Nishio N, Fujimoto Y, Fujii M, Saito K, Hiramatsu M, Maruo $\mathrm{T}$, et al: Craniofacial resection for T4 maxillary sinus carcinoma: managing cases with involvement of the skull base. Otolaryngol Head Neck Surg 153:231-238, 2015

10. Patel MR, Stadler ME, Snyderman CH, Carrau RL, Kassam AB, Germanwala AV, et al: How to choose? Endoscopic skull base reconstructive options and limitations. Skull Base 20:397-404, 2010

11. Patel MR, Taylor RJ, Hackman TG, Germanwala AV, SasakiAdams D, Ewend MG, et al: Beyond the nasoseptal flap: outcomes and pearls with secondary flaps in endoscopic endonasal skull base reconstruction. Laryngoscope 124:846-852, 2014

12. Rastatter JC, Walz PC, Alden TD: Pediatric skull base reconstruction: case report of a tunneled temporoparietal fascia flap. J Neurosurg Pediatr 17:371-377, 2016

13. Saito K, Fukuta K, Takahashi M, Tachibana E, Yoshida J: Management of the cavernous sinus in en bloc resections of malignant skull base tumors. Head Neck 21:734-742, 1999

14. Saito K, Takahashi M, Fukuta K, Tachibana E, Yoshida J: Recovery of olfactory function after an anterior craniofacial approach. Skull Base Surg 9:201-206, 1999

15. Terasaka S, Sawamura Y, Goto S, Fukushima T: A lateral transzygomatic-transtemporal approach to the infratemporal fossa: technical note for mobilization of the second and third branches of the trigeminal nerve. Skull Base Surg 9:277287, 1999
16. Vaezi A, Cardenas E, Pinheiro-Neto C, Paluzzi A, Branstetter BF IV, Gardner PA, et al: Classification of sphenoid sinus pneumatization: relevance for endoscopic skull base surgery. Laryngoscope 125:577-581, 2015

17. Wang J, Bidari S, Inoue K, Yang H, Rhoton A Jr: Extensions of the sphenoid sinus: a new classification. Neurosurgery 66:797-816, 2010

18. Wanibuchi M, Murakami G, Yamashita T, Minamida Y, Fukushima T, Friedman AH, et al: Midsubtemporal ridge as a predictor of the lateral loop formed by the maxillary nerve and mandibular nerve: a cadaveric morphological study. Neurosurgery 69 (1 Suppl Operative):ons95-ons98, 2011

19. Woodworth BA, Palmer JN: Spontaneous cerebrospinal fluid leaks. Curr Opin Otolaryngol Head Neck Surg 17:59-65, 2009

20. Woodworth BA, Prince A, Chiu AG, Cohen NA, Schlosser RJ, Bolger WE, et al: Spontaneous CSF leaks: a paradigm for definitive repair and management of intracranial hypertension. Otolaryngol Head Neck Surg 138:715-720, 2008

\section{Disclosures}

The authors report no conflict of interest concerning the materials or methods used in this study or the findings specified in this paper.

\section{Author Contributions}

Conception and design: Iwami, Saito. Acquisition of data: Iwami, Fujii, Kishida, Jinguji, Yamada, Nishio, Takanari. Analysis and interpretation of data: Iwami, Fujii. Drafting the article: Iwami, Fujii, Bakhit. Critically revising the article: Fujimoto, Ogawa, Kamei, Saito. Reviewed submitted version of manuscript: Iwami, Fujii. Approved the final version of the manuscript on behalf of all authors: Iwami. Statistical analysis: Iwami.

\section{Correspondence}

Kenichiro Iwami: Aichi Medical University, Nagakute City, Aichi Prefecture, Japan. iwamins@gmail.com. 